\title{
INVESTIGACIONES
}

\section{Variables influyentes en la conducta ambiental en alumnos de unidades educativas, región de Coquimbo-Chile}

\author{
Variables affecting the behavior of students \\ in educative units, Región de Coquimbo-Chile
}

\author{
Felicindo Cortes,${ }^{a}$ Ricardo Cabana Villca,${ }^{a b}$ Domingo Vega Toro, ${ }^{a c}$ \\ Héctor Aguirre Sarmiento, ${ }^{d}$ Roberts Muñoz Gómez ${ }^{e}$

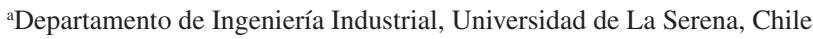 \\ Telf.: (56) 512204195. Correo electrónico: fcortes@userena.cl \\ 'Telf.: (56) 512204197. Correo electrónico: rcabana@userena.cl \\ cTelf.: (56) 512204646. Correo electrónico: dvega@userena.cl
}

${ }^{\mathrm{d}}$ Ejecutivo Empresarial, Fomento Productivo, Municipalidad de Coquimbo, Chile Telf.: (56) 512312771. Correo electrónico: haguirre.investigacion.dii.uls@ gmail.com

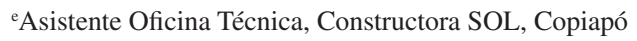

Telf.: (56) 976103205. Correo electrónico: rmunoz.investigacion.dii.uls@gmail.com

\section{RESUMEN}

Este trabajo tiene como propósito desarrollar un modelo sistémico que explique y permita analizar la conducta ambiental en alumnos de establecimientos educacionales certificados, Región Coquimbo-Chile. Se crea una relación entre cada variable que estructura el modelo conductual exploratorio propuesto, con método de Mínimos Cuadrados Parciales (PLS). Para el análisis conductual, se realiza una encuesta con muestra significativa estadísticamente (7\% error y $95 \%$ confiabilidad) en establecimientos educacionales certificados ambientalmente, de las ciudades de Coquimbo y La Serena. El modelo estructural tiene una correcta calidad de predicción, el Test de Stone-Geisser (Q2>0), complementariamente la varianza explicada de cada una de las relaciones es aceptable. En consecuencia, se concluye que las actitudes y los amigos de los mismos estudiantes influyen significativa y directamente en su conducta ambiental. Mientras que las estrategias y valores corporativos de los establecimientos educacionales y la familia no tienen una influencia significativa.

Palabras clave: estrategias, actitudes, valores, ecuaciones estructurales.

\begin{abstract}
The purpose of this paper is to develop a systematic model to explain and analyze the environmental behavior of students from certified educational instituitons in Región de Coquimbo, Chile. A relationship among variables has been established to structure the exploratory behavioral model presented through a Partial Least Squares (PLS) method. For behavioral analysis, a survey was implemented showing a significant statistical sample (7\% error and $95 \%$ reliability) of environmentally certified educational institutions. The structural model presents an appropriate prediction quality, Stone-Geisser test Q2 $>0$; additionaly, the variance explained in each relation is adequate. Consequently, it is concluded that attitude and friendship among students themselves affect the environmental behavior significantly and directly. While corporate startegies and values from insitutions and the family do not seem tho affect crucially.
\end{abstract}

Key words: strategies, attitudes, values, structural equation. 


\section{INTRODUCCIÓN}

En la actualidad, el planeta vive su peor situación ambiental y una de sus principales vías de solución viene de la mano de la educación, en este caso la educación ambiental. Esta es una herramienta fundamental para lograr un cambio de actitud y de comportamiento en la sociedad, no solo en los productores, sino también en los consumidores de todo el mundo (Guzmán \& Fernández, 2013).

De acuerdo con Moyano, Encina y Vicente (2007), las investigaciones que evalúan los impactos de la educación ambiental (EA), parecen confirmar que la EA "mejora la calidad de la educación en general, el rendimiento académico y las habilidades de la ciudadanía" (p. 4), es decir, tienen un efecto positivo sobre el proceso de aprendizaje en sí. Sin embargo, la mayoría de los programas educativos ambientales están caracterizados por proporcionar una gran cantidad de conocimientos científicos, acerca de los problemas ambientales, así como causas y efectos sobre el bienestar de la sociedad, más que sobre procesos formativos (Jensen \& Schnack, 2006; Tsevreni, 2011). De hecho, pocos programas se centran en utilizar metodologías que fomenten el conocimiento a través de la exploración, el contacto directo con las problemáticas asociadas y que estimulen el análisis y reflexión crítica acerca de su entorno (Breiting et al., 2009). La educación ambiental debería incluir las características de la pedagogía crítica basada en el aprendizaje activo y experiencial, así como una reflexión crítica; se debería estimular a los niños para que expresen y comuniquen sus experiencias, ideas y emociones acerca de su entorno y vida diaria (Barratt, Barratt \& Scott, 2007).

El diseño de la nueva institucionalidad ambiental en Chile, releva la educación ambiental como un elemento de vital importancia en el camino hacia la sustentabilidad. En este marco el Ministerio del Medio Ambiente acelera la implementación de programas que busquen fortalecer la educación ambiental, profundizando programas como el Sistema Nacional de Certificación Ambiental en Establecimientos Educacionales (SNCAE) y políticas que estimulan a las universidades a incorporar en su gestión educativa el concepto de "campus sustentable"; al mismo tiempo, se dará un impulso en la educación no formal, propiciando un mayor involucramiento de la ciudadanía en la conservación, preservación y cuidado del medio ambiente (Ministerio del Medio Ambiente, 2010).

Tanto en Sudamérica como en América Latina, se han efectuado investigaciones donde se abordan tanto la conducta como el interés en los problemas ambientales, y se considera que el nivel de conocimiento y la información sobre la temática, determinan las acciones en favor del medio ambiente (Sánchez, Garza-González \& Rodríguez, 2014). Por su parte Barriero, López, Losada y Ruzo (2002), al estudiar los factores que intervienen en el comportamiento ambiental, deducen que las personas que poseen un mayor conocimiento sobre los aspectos ecológicos, son los que manifiestan mayor preocupación por dichos aspectos ecológicos.

Para el desarrollo de esta investigación, se revisa la literatura existente, con el propósito de establecer las variables influyentes en la conducta ambiental, se realiza un trabajo recopilatorio de datos en establecimientos educacionales certificados ambientalmente pertenecientes a las ciudades de Coquimbo y La Serena, para su análisis se formulan y contrastan hipótesis, se establece un modelo de ecuaciones estructurales y se efectúan propuestas para mejorar la conducta ambiental en los establecimientos educacionales estudiados. 


\section{Los valores y las creencias}

Los valores son estructuras del pensamiento que se mantienen pre configuradas en el cerebro de cara a nuestra supervivencia como especie humana, según lo establece Prieto (2011). Martí, Martí-Vilar y Almerich (2014), desde una mirada de la psicología básica propone que los valores subyacen como procesos psicológicos que conducen a ejercer una responsabilidad social.

Miranda (2013) orienta que los valores sirven para establecer relaciones entre las prioridades valorativas y los comportamientos, por ello, conocer como estos se organizan $\mathrm{y}$ articulan puede ofrecer pistas del modo en que se produce un comportamiento proambiental. A su vez, referencia que el origen de los valores está dado desde tres necesidades o requerimientos universales a los que todo individuo o sociedad debe responder: 1) Las necesidades propias de los seres humanos como organismos biológicos; 2) Los requerimientos de acciones sociales coordinadas; 3) Las necesidades de supervivencia y bienestar de los grupos. Además, indica que el problema del constante deterioro ambiental y social se ha atribuido a ciertas creencias o formas de ver el mundo, que establecen en gran parte los valores y las actitudes con respecto al medio ambiente y a los otros seres humanos que generan determinados comportamientos o conductas ecológicas.

El grado de adhesión a creencias favorables a la protección y respeto del medio ambiente, es de especial relevancia, dado que las creencias configuran las verdades básicas sobre la realidad física y social, y la naturaleza de uno mismo; siendo parámetros que usamos para darle sentido a nuestro mundo, se observan como hechos (Murray, 2011).

Según el modelo Valores-Creencias-Normas (Stern, 1999), se postula que los valores influyen en las creencias generales que el individuo tiene sobre el medio ambiente, y según estas creencias el individuo puede ser una persona consciente de las consecuencias que su conducta podría desencadenar en el medio ambiente.

Hipótesis 1: Los valores influyen indirectamente en la conducta ambiental por medio de las creencias.

\section{Las creencias y las actitudes}

Las actitudes son la valoración que hace cada individuo de un estímulo como favorable o desfavorable, es la posición, es la percepción, la forma de interpretar nuestra realidad. Por ello las actitudes son modificables, pueden cambiarse, pueden reevaluarse a través de las experiencias y de la crítica de cada persona: son educables (Prieto, 2011).

Las formas más elaboradas de estructuras sociales generan tipos de conciencia social que, junto a la acción de los medios de comunicación que informan y persuaden respecto de determinados patrones de interacción, conforman un conjunto de creencias, ideas y conocimientos respecto de los recursos ambientales, sea de sus problemáticas y/o valoración (Sandoval, 2012). Según Pérez (2013), las acciones de intervención ambiental, dependen de las creencias de las personas respecto a si poseen o no conocimientos sobre la acción ambiental, si poseen o no la habilidad para ejecutarla y de sus actitudes.

En Chile, según Ministerio del Medio Ambiente (2013), se realizó un estudio sobre "Comportamiento Ambiental de la Ciudadanía" concluyendo que las creencias asocian un objeto con ciertos atributos, es decir, con otros objetos, características o eventos, en el caso 
de las actitudes, los individuos asocian sus creencias con un resultado positivo o negativo, entonces el individuo adquiere automáticamente una actitud hacia un comportamiento.

Las reflexiones de Álvarez y Vega (2010), respecto a la necesidad de instalar en los estudiantes creencias y actitudes positivas hacia el medio ambiente, establecen que: "Una de las posibles razones de las diferencias entre las actitudes y comportamientos es que el interés generalizado por el medio ambiente coexiste con un sentimiento de desesperanza e incapacidad para convertir el interés en acciones" (p. 3568).

Hipótesis 2: Las creencias ambientales influyen indirectamente por medio de la actitud en la conducta ambiental.

\section{Los conocimientos y la motivación ambiental}

Tradicionalmente la motivación se ha entendido como algo situado dentro de la persona que podría explicar algunos comportamientos y tener un papel causal sobre la conducta manifiesta del cambio (Froján et al., 2010). El aprendizaje es un proceso que puede ser conceptuado de forma sintética, es el modo en que las personas adquieren nuevos conocimientos, desarrollando competencias y cambiando el comportamiento (Baracho da Silva, 2010).

Conocer el comportamiento ambiental de las personas y la realidad que lo rodea constituye un factor básico para mejorar sus prácticas y contribuir a la preservación del entorno en la búsqueda de alcanzar la calidad ambiental, en este sentido, educar ambientalmente, es fundamental para que el individuo se comprometa con el cuidado de su ambiente y lo valore, movilizando la conciencia ambiental hasta lograr incorporar la variable ambiental en la toma de decisiones de la persona, tanto en el ámbito personal-familiar, como en el contexto escolar, laboral y los demás ambientes en donde se desenvuelva (Pérez, 2013).

El conocimiento en educación ambiental posibilita disminuir los daños, al tiempo que favorece el logro de una motivación ambiental (Secretaría de ambiente y desarrollo sustentable de la nación, Argentina, 2012). Cheng y Monroe (2012) añaden la importancia de experiencias pasadas en la naturaleza, la proximidad del lugar de residencia a la naturaleza, el conocimiento ambiental como predictores de la motivación de participar de actividades ambientalmente responsables.

Hipótesis 3: Los conocimientos ambientales influyen indirectamente en la conducta ambiental por medio de la motivación.

\section{La motivación ambiental y las actitudes}

La motivación es un estímulo que mueve a la persona a realizar determinadas acciones y persistir en ellas para su culminación, impulsar a alguien a iniciar acciones, encaminadas a conseguir objetivos, y persistir en el intento hasta alcanzarlos (Baracho da Silva, 2010). El concepto de motivación, además se utiliza para explicar las diferencias en la intensidad del comportamiento, los más intensos son considerados como el resultado de niveles más altos de motivación y viceversa (Astudillo \& Leiva, 2010).

El grado de motivación medioambiental auto determinada, señala de forma positiva e indirecta, a través de la actitud, el grado de respeto con el medio ambiente y se considera en la actualidad como uno de los factores que explican el comportamiento ecológico, según lo planteado por Martínez (2012). 
Dada una problemática ambiental, los individuos solo ejecutan conductas proambientales cuando están motivados, se ven capaces de generar cambios y están convencidos de que su acción tendrá efectividad, dicha actitud se ve reforzada cuando dichas actuaciones no les generará dificultades (Miranda, 2013).

Hipótesis 4: La motivación influye indirectamente por medio de la actitud en la conducta ambiental.

\section{Las actitudes y la conducta ambiental}

Las actitudes pro ambientales pueden ser entendidas como aquel comportamiento humano, ya sea individual o colectivo, que busca de manera consciente, proteger, preservar y/o minimizar el impacto negativo sobre el ambiente natural y construido (Corral-Verdugo, Frías \& García, 2010).

Según Miranda (2013), se plantea que las actitudes tienen una gran influencia sobre el comportamiento cuando otros factores no impiden que este se lleve a cabo, sobre todo en lo referente a los comportamientos individuales de consumo y participación ambiental. El análisis de las actitudes ambientales permite identificar los componentes culturales, simbólicos y cognitivos, que sustentan los patrones de interacción sociedad-naturaleza y orientan los usos de los recursos (López, M. 2010).

La crisis medioambiental y la consiguiente toma de conciencia de la importancia de proteger y respetar el entorno natural, requiere un cambio de actitudes en las relaciones entre las personas y su entorno natural, que se traduce en la consideración de la naturaleza por su valor intrínseco y no solo por su utilidad en la mejora de la calidad de vida humana, así como en la incorporación de la naturaleza en el concepto de sí mismo (Amérigo, García \& Sánchez, 2013).

En investigaciones se ha encontrado que cuando los estudiantes tienen educación o cursos sobre el medio ambiente tienen actitudes ambientales mayores que los otros alumnos (Müderrisoğlu \& Altanlar, 2011). A su vez, estudiantes con mayor conocimiento sobre el medio ambiente o mayores actitudes ambientales reportan mayores conductas ambientales (Heyl, 2012). Además, se ha observado que los alumnos presentan mayores actitudes al final de los últimos años en comparación a los primeros, o sea, alumnos que poseen más educación formal presentan actitudes ambientales mejores que aquellos con menor educación formal (Zarrintaj et al., 2011).

Hipótesis 5: Las actitudes ambientales tienen una relación directa con la conducta ambiental.

\section{La familia y la conducta ambiental}

En la familia, un menor se manifiesta y comporta de acuerdo a lo que observa y aprende de sus mayores, por lo tanto, se debe realizar un énfasis en la concepción de valores, los cuales permiten al ser humano transformar su propia realidad desde su propia visión personal y colectiva. Este concepto, se evidencia en la influencia que tiene el núcleo familiar en el menor frente al cuidado, manejo y protección de los recursos naturales, por ello, es importante tener un adecuado proceso de educación ambiental en el hogar (núcleo familiar), para que el niño sea un ser humano que adquiera, aprenda y consolide comportamientos aceptados por la sociedad en función de la protección de los recursos naturales (Galvis, 2009). 
Cada familia muestra a sus miembros lo que se espera de ellos, condicionada principalmente por las directrices y requerimientos culturales provenientes del sistema social en el que se desenvuelve (valores culturales, creencias, sucesos históricos, familia extensa, trabajo, amistades). Tanto padres como hijos interpretan su propia conducta y la del otro, en función de esquemas cognitivos-motivacionales, transmitidos por valores culturales dominantes (Penas, 2008).

El ámbito de la familia se destaca por producir innumerables aprendizajes desde las etapas iniciales del desarrollo. Al respecto, Matthies, Selge y Klöckner (2012) han investigado, entre otros aspectos, la influencia de los padres en los comportamientos pro ambiental de los niños alemanes, considerando la transferencia de normas subjetivas referentes a las consecuencias de dichos comportamientos al medio ambiente. Los resultados más destacados apuntan hacia la relación directa entre el comportamiento de los padres y su influencia en el comportamiento de los hijos.

Hipótesis 6: La familia influye directamente en la conducta ambiental.

\section{Los amigos y la conducta ambiental}

El creciente desarrollo físico y cognitivo que experimentan los adolescentes les permite enfrentarse a las nuevas exigencias que se les presentan a nivel personal, familiar, escolar y social, en dicho proceso, su mundo social rebasará los límites de la familia para encontrarse con sus iguales. Las experiencias personales, escolares y sociales de estos años serían incomprensibles sin tener en cuenta la presencia de sus iguales (Rubín, 1998).

A medida que las personas maduran, sus interacciones sociales se van haciendo más sólidas e intensas, los jóvenes no son ajenos a este fenómeno. El grupo de iguales ayuda a cada uno de sus miembros a desarrollar sentimientos de identidad y pertenencia, influye en su auto concepto y contribuye a la adquisición de competencias personales, pero para los jóvenes es tan importante sentirse aceptados por el grupo, que muchos de sus comportamientos estarán influenciados por esos deseos de sentirse integrados en el grupo. Los amigos son el segundo grupo social, después de la familia, es un espacio privilegiado en la transmisión de valores pues los jóvenes sienten una mayor libertad, al establecer unas relaciones más horizontales, menos formalizadas que permiten experimentar y descubrir el mundo que les rodea, por lo mismo, relacionarse con un grupo donde se practican conductas pro ambientales influirá en la persona en desarrollar también este tipo de conductas (Penas, 2008).

Hipótesis 7: Los amigos tienen directa relación con la conducta ambiental.

\section{Los valores del establecimiento educacional y la conducta ambiental}

La estructura de valores que posee el individuo y en particular los valores a los que se asigna una mayor o menor importancia juegan un papel determinante en la toma de decisiones. Identificar los valores prevalecientes en los directivos al momento de guiar su actividad gerencial, la correspondencia entre la estructura de estos valores y los valores corporativos es de gran importancia, puesto que los valores en la organización permiten que sus integrantes interactúen de manera armónica e inciden en el éxito y crecimiento de dichas organizaciones, en especial cuando la ventaja competitiva se sustenta en la reputación constituida tras largos años de trabajo (Dussan, 2012). 
Obregón (2011) expresa que entre las razones que fundamentan la necesidad de otorgarle una importancia relevante a los valores en el plano gerencial se señalan las siguientes: los valores son los impulsores principales en la actuación de las personas y las organizaciones, son los que otorgan cohesión y sentido de pertinencia, establecen compromisos éticos entre sus miembros y de la organización con sus clientes y socio.

Hipótesis 8: Los valores del establecimiento educacional tienen una relación directa con la conducta ambiental.

\section{Las estrategias de enseñanza y la conducta ambiental}

Se entiende por estrategias de enseñanza al conjunto de decisiones que toma el docente para orientar la enseñanza con el fin de promover el aprendizaje de los alumnos. Se trata de orientaciones acerca de cómo enseñar un contenido disciplinar considerando qué queremos que los alumnos comprendan, por qué y para qué (Anijovich \& Mora, 2010). Ciertas investigaciones realizadas, sugieren analizar cómo utilizamos las imágenes para construir y reforzar la memoria ambiental, la naturaleza es un espectáculo visual ante el cual nuestros ojos necesariamente se detendrán y se deslumbrarán (Hollman, 2012).

Existen tres factores que afectan negativamente la gestión escolar: 1) la ausencia de liderazgo efectivo de quienes dirigen estas organizaciones, 2) un desconocimiento de las herramientas de gestión y 3) una cultura organizacional fuertemente arraigada y opuesta a los cambios organizacionales. Lo indicado genera una resistencia que impide atacar las verdaderas causas de una gestión escolar deficiente (López, P. 2010).

En variados encuentros y cumbres internacionales, se han adquirido múltiples compromisos por parte de los Estados para redefinir sus programas; estos compromisos contemplan la variable ambiental y desarrollan estrategias efectivas de educación ambiental, como uno de los instrumentos para modificar sustancialmente la relación de la sociedad con la naturaleza (Medina \& Páramo, 2014).

La educación ambiental se inscribe como la estrategia actual de cómo abordar la problemática ambiental, más allá de plantear solo respuestas eminentemente técnicas o tecnológicas, lo que hoy se proyecta es un abordaje más integrador, que permita contextualizar los nuevos problemas medioambientales como aspectos claves del desarrollo sostenible en los ámbitos locales (Reyes \& Cardona, 2015).

Hipótesis 9: Las estrategias de enseñanza en los establecimientos educacionales se relacionan directamente con la conducta ambiental.

\section{MÉTODOS}

La metodología estadística utilizada para analizar las hipótesis propuestas es el modelo de ecuaciones estructurales (Structural Equation Modeling, SEM) que permite examinar simultáneamente una serie de relaciones de dependencia y es particularmente útil cuando una variable dependiente se convierte en variable independiente en ulteriores relaciones de dependencia. Además, muchas de las mismas variables afectan a cada una de las mismas variables dependientes, pero con efectos distintos (Hair et al., 1995).

La información utilizada para contrastar las hipótesis propuestas fue obtenida a partir de la aplicación de una encuesta a alumnos pertenecientes a colegios certificados 
ambientalmente (SNCAE) de Coquimbo y La Serena. El tamaño conocido de la población es 6.717 estudiantes, según la información entregada por el Ministerio de Educación, con certificación vigente a febrero del año 2015. A continuación, se presenta la ficha técnica de la investigación (Tabla 1).

Tabla 1. Ficha Técnica de la investigación

\begin{tabular}{|c|c|c|c|}
\hline Colegio & $\mathrm{N}^{\circ}$ de Alumnos & Nivel & Nivel de Certificación ambiental \\
\hline Claudio Arrau & 41 & $3^{\circ}$ y $4^{\circ}$ medio & Medio \\
\hline Sta. María del Refugio & 42 & $7^{\circ}$ y $8^{\circ}$ básico & Básico \\
\hline Saint Johns School & 143 & $7^{\circ}$ y $8^{\circ}$ básico & Básico \\
\hline \multicolumn{2}{|c|}{ Tamaño muestral encuestado } & \multicolumn{2}{|r|}{226} \\
\hline \multicolumn{2}{|c|}{ Unidad de análisis } & \multicolumn{2}{|r|}{ Alumnos } \\
\hline \multicolumn{2}{|l|}{ Tipo de muestreo } & \multicolumn{2}{|r|}{ Probabilístico } \\
\hline \multicolumn{2}{|l|}{ Nivel de Confianza } & \multicolumn{2}{|c|}{$95 \% ; \mathrm{z}=1,96 \mathrm{p}=\mathrm{q}=0,5(7 \%$ de error $\mathrm{y} 95 \%$ confiabilidad $)$} \\
\hline \multicolumn{2}{|l|}{ Técnicas estadísticas } & \multicolumn{2}{|c|}{$\begin{array}{l}\text { Análisis descriptivo } \\
\text { Análisis Causal PLS }\end{array}$} \\
\hline \multirow{10}{*}{\multicolumn{2}{|c|}{ Ítem Instrumento de Evaluación }} & \multicolumn{2}{|c|}{ Ítem 1: Conducta Ambiental (3 preguntas) } \\
\hline & & \multicolumn{2}{|c|}{ Ítem 2: Valores (4 preguntas) } \\
\hline & & \multicolumn{2}{|c|}{ Ítem 3: Creencias (4 preguntas) } \\
\hline & & \multicolumn{2}{|c|}{ Ítem 4: Conocimientos (4 preguntas) } \\
\hline & & \multicolumn{2}{|c|}{ Ítem 5: Actitudes (3 preguntas) } \\
\hline & & \multicolumn{2}{|c|}{ Ítem 6: Motivación (4 preguntas) } \\
\hline & & \multicolumn{2}{|c|}{ Ítem 7: Amigos (3 preguntas) } \\
\hline & & \multicolumn{2}{|c|}{ Ítem 8: Familia (4 preguntas) } \\
\hline & & \multicolumn{2}{|c|}{$\begin{array}{l}\text { Ítem 9: Estrategia Establecimiento Educacional } \\
\text { (5 preguntas) }\end{array}$} \\
\hline & & \multicolumn{2}{|c|}{$\begin{array}{l}\text { Ítem 10: Valores Establecimiento Educacional } \\
\text { (4 preguntas) }\end{array}$} \\
\hline \multicolumn{2}{|c|}{ Tipo de pregunta encuesta } & \multicolumn{2}{|c|}{ 100\% politómicas tipo Likert } \\
\hline \multicolumn{2}{|c|}{ Fecha de realización del trabajo de campo } & \multicolumn{2}{|c|}{25 de mayo a 19 de junio del año 2015} \\
\hline
\end{tabular}

Fuente: elaboración propia.

\section{RESULTADOS}

Para el análisis estadístico se utiliza el software SPSS Statics 20 y SmartPLS 2.0.

\section{Fiabilidad Individual de los Indicadores}

Para determinar la fiabilidad individual se obtuvo la carga factorial individual de cada uno de los indicadores señalados en la encuesta, esto fue posible gracias a que el índice de 
Kaiser-Meyer-Olkin (KMO) fue superior a 0,5 y la prueba de esfericidad de Bartlett (PEB) resultó ser significativa $(\mathrm{p}<0,05)$ para cada uno de los constructos (Méndez \& Rondon, 2012). Se eliminan los indicadores CRE1, CONO4, MOT1 y FAM4, no cumplen carga factorial mínima aceptable 0,7 (Barclay, Higgins \& Thompson, 1995). Los resultados se detallan en la Tabla 2.

Tabla 2. Cargas Factoriales de los Indicadores

\begin{tabular}{|c|c|c|}
\hline Constructo & Indicador & Carga Factorial \\
\hline \multirow{3}{*}{$\begin{array}{l}\text { Conducta Ambiental } \\
\mathrm{KMO}=0,754 \\
\mathrm{PEB}=0,00(\mathrm{p}<0,05)\end{array}$} & COND1: Comunicar temas ambientales & 0,917 \\
\hline & COND2: Satisfacción acciones pro ambientales & 0,930 \\
\hline & COND3: Sacrificios personales & 0,912 \\
\hline \multirow{4}{*}{$\begin{array}{l}\text { Valores } \\
\mathrm{KMO}=0,799 \\
\mathrm{PEB}=0,00(\mathrm{p}<0,05)\end{array}$} & VAL1: TV encendida solo si es necesario & 0,807 \\
\hline & VAL2: Llave agua abierta solo si es necesario & 0,877 \\
\hline & VAL3: Tiempo ducha corto & 0,854 \\
\hline & VAL4: Molestia por basura esparcida & 0,882 \\
\hline \multirow{4}{*}{$\begin{array}{l}\text { Creencias } \\
\mathrm{KMO}=0,777 \\
\mathrm{PEB}=0,00(\mathrm{p}<0,05)\end{array}$} & CRE1: Preocupación escasez de agua & 0,396 \\
\hline & CRE2: Molestia uso de agua & 0,939 \\
\hline & CRE3: Reciclaje & 0,938 \\
\hline & CRE4: Preocupación consumo energía & 0,921 \\
\hline \multirow{4}{*}{$\begin{array}{l}\text { Conocimientos } \\
\mathrm{KMO}=0,781 \\
\mathrm{PEB}=0,00(\mathrm{p}<0,05)\end{array}$} & CONO1: Valor de la basura & 0,935 \\
\hline & CONO2: Clasificación desechos & 0,922 \\
\hline & CONO3: Definición reciclaje & 0,933 \\
\hline & CONO4: Separación desechos & 0,398 \\
\hline \multirow{3}{*}{$\begin{array}{l}\text { Actitudes } \\
\mathrm{KMO}=0,755 \\
\mathrm{PEB}=0,00(\mathrm{p}<0,05)\end{array}$} & ACT1: Intención participación & 0,925 \\
\hline & ACT2: Intención unirse a un grupo & 0,915 \\
\hline & ACT3: Intención ayudar a organización & 0,914 \\
\hline \multirow{4}{*}{$\begin{array}{l}\text { Motivación } \\
\mathrm{KMO}=0,757 \\
\mathrm{PEB}=0,00(\mathrm{p}<0,05)\end{array}$} & MOT1: Informarse temas ambientales & 0,389 \\
\hline & MOT2: Consumo producto dañinos & 0,930 \\
\hline & MOT3: Utilización papel reciclado & 0,926 \\
\hline & MOT4: Reducción consumo luz & 0,912 \\
\hline \multirow{3}{*}{$\begin{array}{l}\text { Amigos } \\
\mathrm{KMO}=0,742 \\
\mathrm{PEB}=0,00(\mathrm{p}<0,05)\end{array}$} & AMI1: Promover cuidado ambiental & 0,904 \\
\hline & AMI2: Cuidado medio ambiente & 0,890 \\
\hline & AMI3: Interés medio ambiente & 0,919 \\
\hline
\end{tabular}


Estudios Pedagógicos XLIII, N² 2: 27-46, 2017

VARIABLES INFLUYENTES EN LA CONDUCTA AMBIENTAL EN ALUMNOS DE UNIDADES EDUCATIVAS, REGIÓN DE COQUIMBO-CHILE

\begin{tabular}{|l|l|l|}
\hline \multirow{4}{*}{$\begin{array}{l}\text { Familia } \\
\text { KMO }=0,754 \\
\text { PEB }=0,00(\mathrm{p}<0,05)\end{array}$} & FAM1: Preocupación reciclaje & 0,896 \\
\cline { 2 - 3 } & FAM2: Beneficio reciclaje & 0,887 \\
\cline { 2 - 3 } & FAM3: Separación basura & 0,906 \\
\cline { 2 - 3 } & FAM4: Reciclaje en casa & 0,323 \\
\hline \multirow{3}{*}{$\begin{array}{l}\text { Estrategia Establec. } \\
\text { Educacional } \\
\text { KMO }=0,859 \\
\text { PEB }=0,00(\mathrm{p}<0,05)\end{array}$} & EST1: Educación & 0,859 \\
\cline { 2 - 3 } \begin{tabular}{l} 
EST2: Educación ambiental \\
\cline { 2 - 3 } $\begin{array}{l}\text { Valores Establec. } \\
\text { Educacional }\end{array}$
\end{tabular} & EST3: Contenido ambiental atractivo & 0,902 \\
\cline { 2 - 3 } $\begin{array}{l}\text { KMO }=0,788 \\
\text { PEB }=0,00(p<0,05)\end{array}$ & EST5: Propuestas ambientales & 0,814 \\
\cline { 2 - 3 } & VEE1: Respeto medio ambiente & 0,861 \\
\cline { 2 - 3 } & VEE3: Cuidado ambiental & 0,846 \\
\cline { 2 - 3 } & VEE4: Basura producida & 0,858 \\
\hline
\end{tabular}

Fuente: elaboración propia.

\section{Fiabilidad del Constructo}

Para evaluar la consistencia interna de los indicadores que miden los constructos reflectivos, se analiza la fiabilidad del constructo mediante la revisión del Alfa de Cronbach y el índice de fiabilidad compuesta (IFC). Al respecto, Oviedo y Campos-Arias (2005) plantean que un valor de Alfa de Cronbach sobre 0,7 indica una buena consistencia interna para una escala unidimensional. Henseler, Ringle y Sinkovics (2009) plantean que el IFC debe tener un valor mínimo de 0,7 para ser aceptada. De acuerdo a lo anterior los valores obtenidos son aceptables para todas las variables estudiadas.

Tabla 3. Alfa de Cronbach, Fiabilidad Compuesta y AVE de los Constructos

\begin{tabular}{|l|c|c|c|}
\hline Constructo & $\begin{array}{c}\text { Alfa de } \\
\text { Cronbach }\end{array}$ & $\begin{array}{c}\text { Fiabilidad } \\
\text { Compuesta }\end{array}$ & AVE \\
\hline Actitudes & 0,905 & 0,9415 & 0,8428 \\
\hline Amigos & 0,888 & 0,9307 & 0,8172 \\
\hline Conducta Ambiental & 0,909 & 0,9427 & 0,8458 \\
\hline Conocimientos & 0,933 & 0,9572 & 0,8817 \\
\hline Creencias & 0,936 & 0,9593 & 0,8872 \\
\hline Estrategia Establecimiento Educacional & 0,908 & 0,9322 & 0,7337 \\
\hline
\end{tabular}




\begin{tabular}{|l|l|l|l|}
\hline Familia & 0,887 & 0,9303 & 0,8164 \\
\hline Motivación & 0,923 & 0,9517 & 0,8679 \\
\hline Valores & 0,874 & 0,9156 & 0,7310 \\
\hline Valores Establecimiento Educacional & 0,875 & 0,9141 & 0,7269 \\
\hline
\end{tabular}

Fuente: elaboración propia.

\section{Validez del Constructo}

Dicho análisis considera la comprobación de la validez convergente y discriminante de los constructos, en el cálculo de dichos indicadores se siguieron los pasos entregados por Henseler et al. (2009).

El análisis de la validez convergente se obtiene a partir del cálculo de la varianza extraída (AVE) para los constructos reflectivos. Un valor AVE de al menos 0,5 indica capacidad del constructo para explicar más de la mitad de la varianza de sus indicadores en promedio (Henseler et al., 2009). Como se puede observar en la Tabla 3, el valor AVE es mayor a 0,5 para todos los constructos, por lo que se comprueba la validez convergente de estos.

Para el análisis de validez discriminante, en Tabla 4 se muestra la matriz de correlaciones de los constructos, habiéndose sustituido en la diagonal el valor de la correlación por la raíz cuadrada de AVE (Henseler et al., 2009).

Al ser los valores de la diagonal los mayores de cada fila y columna, se puede afirmar la existencia de validez discriminante.

Tabla 4. Matriz de Correlaciones de los Constructos

\begin{tabular}{|l|c|c|c|c|c|c|c|c|c|c|}
\hline & Actitudes & Amigos & Conducta & Conocimientos & Creencias & $\begin{array}{c}\text { Estrategia } \\
\text { EE }\end{array}$ & Familia & Motivación & Valores & $\begin{array}{c}\text { Valores } \\
\text { EE }\end{array}$ \\
\hline Actitudes & $\mathbf{0 , 9 1 8}$ & & & & & & & & & \\
\hline Amigos & 0,420 & $\mathbf{0 , 9 0 4}$ & & & & & & & & \\
\hline Conducta & 0,651 & 0,544 & $\mathbf{0 , 9 1 9}$ & & & & & & & \\
\hline Conocimientos & 0,452 & 0,193 & 0,475 & $\mathbf{0 , 9 3 8}$ & & & & & & \\
\hline Creencias & 0,475 & 0,154 & 0,503 & 0,541 & $\mathbf{0 , 9 4 1}$ & & & & & \\
\hline Estrategia EE & 0,483 & 0,322 & 0,514 & 0,352 & 0,361 & $\mathbf{0 , 8 5 6}$ & & & & \\
\hline Familia & 0,445 & 0,511 & 0,494 & 0,296 & 0,261 & 0,393 & $\mathbf{0 , 9 0 3}$ & & & \\
\hline Motivación & 0,566 & 0,238 & 0,465 & 0,479 & 0,492 & 0,382 & 0,2374 & $\mathbf{0 , 9 3 1}$ & & \\
\hline Valores & 0,292 & 0,175 & 0,340 & 0,477 & 0,503 & 0,221 & 0,335 & 0,401 & $\mathbf{0 , 8 5 5}$ & \\
\hline Valores EE & 0,256 & 0,178 & 0,221 & 0,303 & 0,226 & 0,213 & 0,167 & 0,168 & 0,330 & $\mathbf{0 , 8 5 2}$ \\
\hline
\end{tabular}

EE: Establecimiento Educacional Fuente: elaboración propia. 


\section{Modelo de Ecuaciones Estructurales}

Se utiliza el modelo de ecuaciones estructurales a través del Método de Mínimos Cuadrados Parciales (Partial Least Squares, PLS), dado que este método está orientado a modelos de carácter predictivo y exploratorio, en donde el conocimiento teórico no está del todo desarrollado, como es el caso del modelo propuesto en la Figura 1.

Figura 1. Modelo Causal de Conducta Ambiental

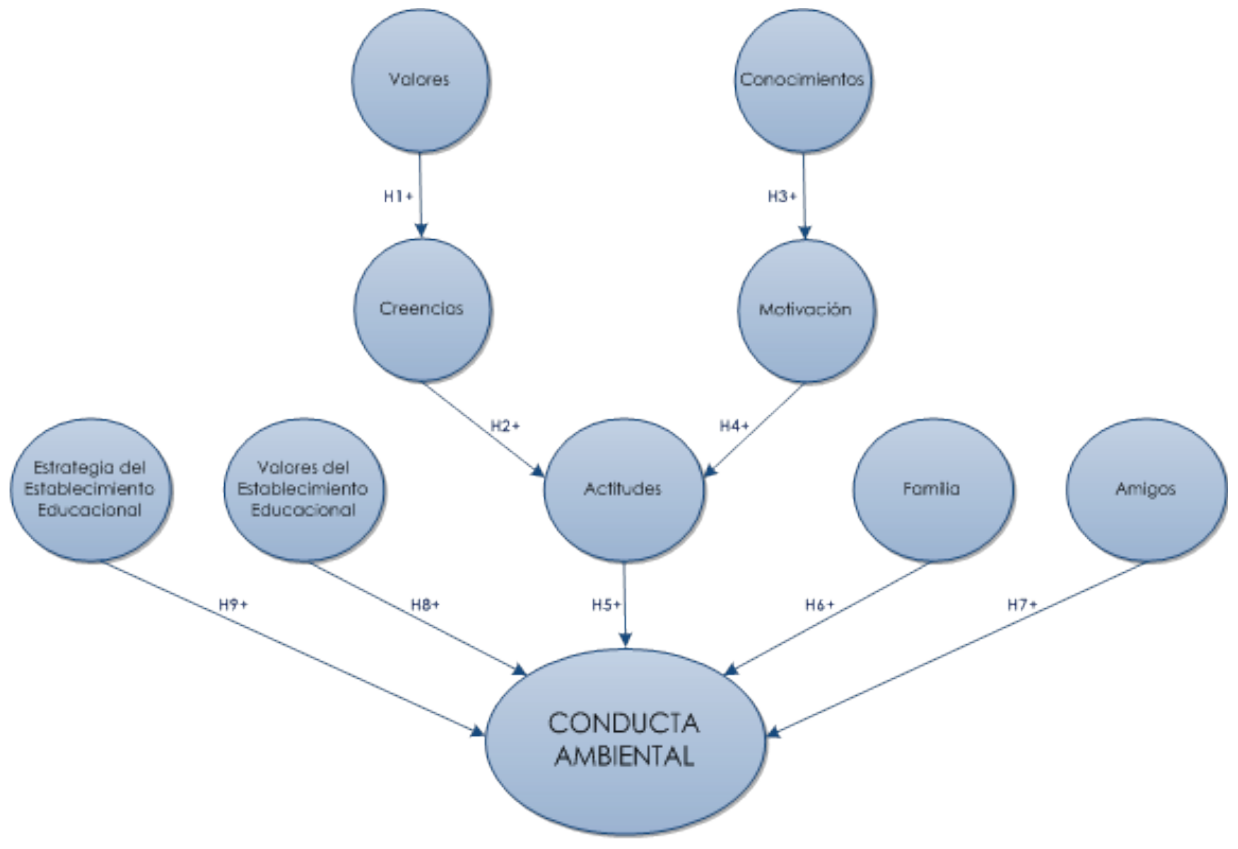

Fuente: elaboración propia.

\section{Validación del modelo estructural}

A continuación, se evalúan las relaciones causales que se proponen en el modelo por medio de dos índices básicos: la varianza explicada $\left(\mathrm{R}^{2}\right)$ y los coeficientes path o pesos de regresión estandarizados $(\beta)$.

La varianza explicada de las variables endógenas o dependientes $\left(\mathrm{R}^{2}\right)$ debe ser igual o mayor que 0,1 (Falk \& Miller, 1992). A partir de este criterio empírico todos los constructos poseen una calidad de poder de predicción aceptable, como se observa en la Tabla 5.

$\mathrm{Al}$ respecto, el valor $\mathrm{R}^{2}$ para la Conducta Ambiental es 0,5559, lo que quiere decir que el $55,59 \%$ de la varianza de este constructo es explicada por el modelo. Entonces, se puede afirmar que el 55,59\% de la varianza de Conducta Ambiental viene determinada por las Actitudes Ambientales, el Grupo de Amigos, el Grupo Familiar, los Valores del Establecimiento Educacional y las Estrategias del Establecimiento Educacional. 
A su vez y aunque sean unos resultados secundarios, se puede comentar que la varianza del constructo Actitudes se explica en un $37,21 \%$ por la Motivación y las Creencias. En tanto, la varianza del constructo Creencias se explica en un 25,70\% por los Valores y la varianza del constructo Motivación se explica en un 22,98\% por los Conocimientos.

Otra prueba que determina la calidad de predicción del modelo estructural es el Test de Stone-Geisser $\left(\mathrm{Q}^{2}\right)$. Este test se usa como criterio para medir la relevancia predictiva de los constructos dependientes y se calcula por medio de la técnica Blindfolding. En el caso de que la $\mathrm{Q}^{2}>0$, indica que el modelo tiene relevancia predictiva. En caso contrario, no la tiene (Chin, 1998).

Como se puede observar en la Tabla 5, en todos los casos los valores de $\mathrm{Q}^{2}$ son positivos, lo cual certifica la relevancia predictiva del modelo.

Tabla 5. Varianza Explicada y Test de Stone-Geisser

\begin{tabular}{|c|c|c|}
\hline Constructo (variable) dependiente) & $\mathrm{R}^{2}$ & $\mathrm{Q}^{2}$ \\
\hline Creencias & 0,2570 & 0,8871 \\
\hline Motivación & 0,2298 & 0,8679 \\
\hline Actitudes & 0,3721 & 0,8428 \\
\hline Conducta Ambiental & 0,5559 & 0,8458 \\
\hline
\end{tabular}

Fuente: elaboración propia.

Los coeficientes path o pesos de regresión estandarizados $(\beta)$, los valores deseables deberían estar por encima de 0,3 siendo 0,2 un valor mínimo aceptable (Chin, 1998). De la Tabla 6, el Paths de las relaciones: Estrategias EE $\rightarrow$ Conducta Ambiental; Familia $\rightarrow$ Conducta Ambiental y Valores EE $\rightarrow$ Conducta Ambiental, no superan el valor mínimo $(0,2)$, las demás relaciones cumplen con la regla de estar sobre el valor mínimo aceptado.

Tabla 6. Coeficientes Paths, Varianza Explicada y Contraste de las Relaciones

\begin{tabular}{|l|c|c|c|c|c|}
\hline \multicolumn{1}{|c|}{ Relaciones } & $\begin{array}{c}\text { Signo de la } \\
\text { hipótesis }\end{array}$ & $\begin{array}{c}\text { Paths } \\
(\beta)\end{array}$ & Correlación & $\begin{array}{c}\text { Varianza } \\
\text { Explicada }\end{array}$ & Contraste \\
\hline H1: Valores $\rightarrow$ Creencias & + & 0,5069 & 0,5069 & $25,69 \%$ & Aceptada \\
\hline H2: Creencias $\rightarrow$ Actitudes & + & 0,2591 & 0,4754 & $12,32 \%$ & Aceptada \\
\hline H3: Conocimientos $\rightarrow$ Motivación & + & 0,4793 & 0,4793 & $22,97 \%$ & Aceptada \\
\hline H4: Motivación $\rightarrow$ Actitudes & + & 0,4392 & 0,5668 & $24,89 \%$ & Aceptada \\
\hline H5: Actitudes $\rightarrow$ Conducta Ambiental & + & 0,3970 & 0,6512 & $25,85 \%$ & Aceptada \\
\hline
\end{tabular}




\begin{tabular}{|l|c|c|c|c|c|}
\hline H6: Familia $\rightarrow$ Conducta Ambiental & + & 0,1096 & 0,4969 & $5,45 \%$ & Rechazada \\
\hline H7: Amigos $\rightarrow$ Conducta Ambiental & + & 0,2582 & 0,5458 & $14,09 \%$ & Aceptada \\
\hline H8: Valores EE $\rightarrow$ Conducta Ambiental & + & 0,0143 & 0,2214 & $0,32 \%$ & Rechazada \\
\hline H9: Estrategia EE $\rightarrow$ Conducta Ambiental & + & 0,1924 & 0,5138 & $9,88 \%$ & Rechazada \\
\hline
\end{tabular}

Fuente: elaboración propia.

Para poder considerar que una relación tiene suficiente capacidad predictiva también se puede analizar la varianza explicada, la cual es el producto de la correlación entre las variables y sus coeficientes paths, la cual según Falk y Miller (1992) debe ser superior a 0,1 (10\%). Entonces, a partir de la Tabla 6 las relaciones H6, H8 y H9 son rechazadas.

\section{Índice de Bondad de Ajuste (GoF)}

Para los modelos estructurales PLS se sugiere un criterio global de bondad de ajuste (Esposito et al., 2008). Estos autores proponen que el índice de bondad de ajuste global se obtenga por medio de la raíz cuadrada de la multiplicación de la media aritmética de la varianza extraída (AVE) de cada constructo y la media aritmética de la varianza explicada $\left(\mathrm{R}^{2}\right)$ de los constructos dependientes.

Según la Tabla 7, el índice de bondad de ajuste (GoF) del modelo propuesto es 0,5369, que representa un buen ajuste en el modelo de medida y en el modelo estructural, cumpliendo así con el criterio empírico de que la medida de bondad de ajuste debe variar entre 0 y 1, a mayor valor, mejor será el índice.

Tabla 7. Índice de Bondad de Ajuste

\begin{tabular}{|l|c|c|c|}
\hline Constructo & AVE & $\mathrm{R}^{2}$ & $\mathrm{GoF}$ \\
\hline Actitudes & 0,8428 & 0,3721 & \\
\hline Amigos & 0,8172 & & \\
\hline Conducta Ambiental & 0,8458 & 0,5559 & \\
\hline Conocimientos & 0,8817 & & \\
\hline Creencias & 0,8872 & 0,2570 & \\
\hline Estrategia Establecimiento Educacional & 0,7337 & & \\
\hline Familia & 0,8164 & & \\
\hline Motivación & 0,8679 & 0,2298 & \\
\hline Valores & 0,7310 & & \\
\hline Valores Establecimiento Educacional & 0,7269 & & \\
\hline \multicolumn{1}{|c|}{ Media Aritmética } & 0,8151 & 0,3537 & 0,5369 \\
\hline
\end{tabular}




\section{DISCUSIÓN}

Los resultados ponen de manifiesto que los Valores influyen de forma significativa en las Creencias (H1: $\beta=0,5069 ; \mathrm{p}>0,01)$, confirmando la relación del modelo Valores $\rightarrow$ Creencias (Stern, 1999), en donde se postula que los valores influyen en las creencias que el individuo tiene, y según estas creencias el individuo puede ser una persona consciente de las consecuencias que su conducta podría desencadenar en el medio ambiente. La constante presencia del tema referido al deterioro ambiental se ha relacionado con ciertas creencias referidas a la manera de ver el mundo, y que a su vez establecen gran parte de los valores con respecto al medio ambiente. Por ello, es relevante fortalecer la "Conducta Ambiental", se debe formar desde tempranas edades una adecuada cultura ambiental, como una alternativa para preservar el medio ambiente, al tiempo que acrecienta la base valórica del individuo (Miranda, 2013).

Las Creencias personales influyen de forma significativa en las Actitudes (H2: $\beta=0,2591 ; p>0,05)$, los individuos asocian sus creencias con un resultado positivo o negativo, entonces el individuo adquiere automáticamente una actitud hacia un comportamiento. La adherencia a creencias ambientales favorece la protección y el respeto hacia el medio ambiente, ya que las creencias configuran las verdades básicas sobre la realidad física y social y la naturaleza de uno mismo (Murray, 2011).

Los Conocimientos que poseen las personas influyen de forma significativa en su Motivación (H3: $\beta=0,4793 ; p>0,01$ ), contribuyen a lograr que los individuos comprendan las complejidades del medio ambiente, la asimilación y comprensión de este conocimiento, generará la motivación que permitirá la participación responsable y eficaz en la prevención y la solución de los problemas ambientales. En esta línea, la creación del Sistema Nacional de Certificación Ambiental de Establecimientos Educacionales (SNCAE) ha permitido desarrollar líneas de acción complementarias, que han logrado fortalecer la educación ambiental, el cuidado y la protección del medio ambiente (Barazarte et al., 2010). Se observa que el aprendizaje es un proceso en el que los estudiantes adquieren nuevos conocimientos, desarrollando competencias y cambiando el comportamiento (Baracho da Silva, 2010), que es relevante para que una unidad educativa obtenga una certificación en la clasificación otorgada por el SNCAE.

La Motivación que tienen las personas influye de forma significativa en las Actitudes (H4: $\beta=0,4392 ; p>0,01$ ), hoy en día la motivación del individuo está siendo considerada como uno de los factores que explican el comportamiento ecológico (Martínez, 2012), puede actuar como barrera o facilitador de la intención del comportamiento, las motivaciones en la protección de la naturaleza parecen mejorar la intención del comportamiento (Barr, 2007).

Las Actitudes influyen de forma significativa en la Conducta Ambiental de los alumnos (H5: $\beta=0,3970 ; p>0,01)$, el análisis de las actitudes ambientales es fundamental para determinar los componentes culturales, simbólicos y cognitivos, que sustentan los patrones de interacción sociedad-naturaleza y a su vez orientan el uso de los recursos (López, M. 2010), cuestión que es influyente en la conducta ambiental que asumirá un alumno.

El Grupo Familiar no influye de forma significativa en la Conducta Ambiental de los alumnos (H6: $\beta=0,1096 ; p>0,05)$, las opiniones que los jóvenes escuchan y aprenden dentro del seno familiar, mediarán en gran medida sobre su forma de entender, filtrar y apreciar las influencias que le ofrecen los demás ámbitos sociales en los que se desenvuelven, esta 
situación no sería equivalente respecto de los contenidos ambientales que son parte de las dinámicas y enseñanzas escolares, pues no estarían recibiendo la importancia que debieran por la mayor parte de las familias (Penas, 2008).

El Grupo de Amigos influye significativamente en la Conducta Ambiental de los alumnos (H7: $\beta=0,2582 ; p>0,01)$, los amigos se encuentran entre las personas más significativas para el adolescente, son las personas con las que pasan más tiempo y comparten sus actividades, con lo cual alcanzan una mayor identidad y pertinencia entre el grupo de iguales, por ello, relacionarse con un grupo donde se practican conductas pro ambiental influirá en las personas en desarrollar también este tipo de conductas (Penas, 2008).

Los Valores del Establecimiento Educacional no influyen de forma significativa en la Conducta Ambiental de los alumnos (H8: $\beta=0,0143$; $p>0,05$ ). A pesar de la eventual presencia de textos escritos, de videos alusivos a la ética e integridad de la corporación, donde se destacan los valores ambientales por parte de los establecimientos educacionales, el real comportamiento de sus integrantes puede ciertamente diferir de lo que marcan tales textos o imágenes. La organización debe asegurarse de que sus valores estén claramente definidos y que cada miembro de la organización adopte estos valores como suyos, esto se puede mostrar cuando lo líderes muestran con hechos cómo se viven los valores de la empresa. La alineación de los valores de la empresa con los valores personales es un punto fundamental para el éxito de este propósito (Dussan, 2012).

La mala transmisión de los valores organizacionales a los alumnos está relacionada directamente con los colaboradores (directivos y docentes), y con el direccionamiento estratégico, es por esto que se debe estar atento a los cambios de comportamiento en los colaboradores y aplicación de los componentes de la cultura organizacional y de cómo se interioriza el direccionamiento estratégico (Quintero, 2010).

Entonces, para que los valores del establecimiento educacional se conviertan en principios de acción, es necesario buscar caminos con una mirada de trabajo cooperativo entre los miembros de la comunidad educacional: directivos, profesores, padres y estudiantes, que lleven a mejorar la conducta ambiental de los alumnos. Como complemento, es necesario mejorar el espacio en el cual se desarrollan las actividades pedagógicas, colocar acento en la importancia y los beneficios que reporta el conservar todo limpio y ordenado, como también respetar y cuidar los recursos naturales con que se cuenta, incrementando así la base valórica de los alumnos.

Las Estrategias de Enseñanza del Establecimiento Educacional no influyen de forma significativa en la Conducta Ambiental de los alumnos (H9: $\beta=0,1924 ; \mathrm{p}>0,05$ ), esto se interpreta como que las estrategias ambientales no se están ejecutando de manera correcta, lograr que las estrategias funcionen es más difícil que diseñarlas, lo que es consistente con lo planteado por Hrebiniak (2005) y Niven (2002). Los colegios están más preocupados de que sus alumnos tengan buenos resultados académicos que de generar cambios en su conducta ambiental, lo que hace que se produzca una falta de compromiso y escasa preocupación en los temas ambientales, lo que obstaculiza la ejecución correcta de las estrategias ambientales (Collins, 2011). No obstante, el valor $\beta$ obtenido en las Estrategias de Enseñanza del Establecimiento Educacional $(\beta=0,1924)$ demuestra que sí puede tener un impacto significativo en la Conducta Ambiental ya que está muy cerca del valor mínimo aceptado $(\beta=0,2)$.

Se requiere cambiar la concepción y el cómo acercarse a la realidad que tienen los alumnos de su entorno, las estrategias de educación ambiental se deben enfocar de acuerdo con las características propias de la comunidad y se hace importante desarrollar estudios en 
los cuales se determinen estas variables o dimensiones, si se quiere avanzar en la búsqueda del progreso y la consolidación de una conducta ambiental favorable con el medio ambiente (Miranda, 2013).

El análisis de los resultados obtenidos sugiere intensificar el trabajo en temáticas ambientales y así alcanzar un mayor impacto en la "Conducta Ambiental" de los alumnos. Para ello se debe contar con líderes sociales y educacionales empoderados, que tengan la convicción de abordar los desafíos ambientales, pues no se está otorgando el sentido de cohesión y pertenencia a los compromisos éticos ambientales (Obregón, 2011).

Se hace necesario potenciar el liderazgo estratégico en organizaciones educacionales que tengan como propósito instalar conductas ambientales en sus alumnos, pues así se posibilitará el logro de una visión donde los valores pro ambiental serán centrales y además se instalarán las capacidades para gestionar con eficacia el entorno. Con esto, se podrán integrar sinérgicamente factores como los Valores del Establecimiento Educacional, las Estrategias de Enseñanza, los Amigos del estudiante y la Familia, que en estas unidades educativas actualmente no tienen una influencia significativa.

\section{CONCLUSIONES}

Las hipótesis formuladas fueron contrastadas, dicho análisis evidencia la existencia de influencias significativas de las distintas variables en estudio, excepto las Estrategia y Valores de los Establecimientos Educacionales y la Familia, cuya influencia no es significativa.

El modelo estructural formulado tiene una correcta calidad de predicción pues el Test de Stone-Geisser tiene métricas $\mathrm{Q}^{2}>0$ y complementariamente la varianza explicada de cada una de las relaciones es aceptable, por tanto, este contribuye en identificar relaciones que influyen en la conducta ambiental de los alumnos y así asociar planes y/o estrategias para mejorar dicha conducta.

Los alumnos poseen los conocimientos suficientes que los hacen tener una motivación mayor por el cuidado del medio ambiente, pero estos conocimientos no necesariamente vienen por parte del establecimiento educacional, más bien obedece a las interrelaciones con sus amistades.

Dado que la influencia de la Familia en los resultados analizados no es significativa, contrario a lo esperado, es necesario realizar más esfuerzos en orden a otorgarle un mayor rol en la formación ambiental de los menores, como eje fundamental en el proceso de enseñanza-aprendizaje, ya que estos adquieren muchos de sus hábitos desde el hogar, en un continuo proceso de socialización e interrelación con su entorno, es necesario desarrollar en los menores los valores ambientales y así dar cumplimiento a la función ecológica que debe ejercer la familia.

\section{REFERENCIAS BIBLIOGRÁFICAS}

Álvarez, P., \& Vega, P. (2010). Developing sustainable environmental behavior in secondary education students (12-16): Analysis of a didactic strategy. Procedia Social and Behavioral Sciences, 2(2), 3568-3574. 
Estudios Pedagógicos XLIII, N² 2: 27-46, 2017

VARIABLES INFLUYENTES EN LA CONDUCTA AMBIENTAL EN ALUMNOS DE UNIDADES EDUCATIVAS, REGIÓN DE COQUIMBO-CHILE

Amérigo, M., García, J., \& Sánchez, T. (2013). Actitudes y comportamiento hacia el medio ambiente natural. Salud medioambiental y bienestar emocional. Universitas Psychologica, 12(3), 845856.

Anijovich, R., \& Mora, S. (2010). Estrategias de enseñanza: otra mirada al quehacer en el aula. Buenos Aires: Aique Grupo Editor.

Astudillo, T., \& Leiva, R. (2010). Factores que influyen en el aprendizaje del idioma inglés en alumnas del Liceo Santa María la Blanca de Valdivia (Tesis para optar al título de Profesor de Comunicación en Lengua Inglesa). Universidad Austral de Chile, Facultad de Filosofía y Humanidades, Valdivia.

Baracho da Silva, A.S. (2010). La relación entre motivación y aprendizaje en el E/ELE. Revista Litteris-Lingüística, 5. Recuperado desde https://www.revistalitteris.com.br/blank-14

Barazarte, R., Neaman, A., Vallejo, F., \& García, E. (2014). El conocimiento ambiental y el comportamiento proambiental de los estudiantes de la enseñanza media, en la región de Valparaíso (Chile). Revista de Educación, Pontificia Universidad Católica de Valparaíso, 364, 12-34. doi:10.4438/1988-592X-RE-2014-364-255

Barclay, D., Higgins, C., \& Thompson, R. (1995). The Partial Least Squares (PLS) Approach to Causal Modelling: Personal Computer Adoption and Use as an Illustration. Technology Studies, Special Issue on Research Methodology, 2(2), 285-309.

Barr, S. (2007). Factors influencing environmental attitudes and behaviors - A UK case study of household waste management. Environment and Behavior, 39(4), 435-473.

Barratt, E., Barratt, R., \& Scott, W. (2007). Engaging Children: Research Issues Around Participation and Environmental Learning. Environmental Education Research, 13, 529-544.

Barriero, J., López, M.A., Losada, P.F., \& Ruzo, E. (2002). Análisis de las dimensiones cognoscitivas y afectivas del comportamiento ecológico del consumidor. Revista Gallega de Economía, 11(2), 1-21.

Breiting, S.K., Hedegaard, K., Mogensen, F., Nielsen, K., \& Schnack, K. (2009). Action competence, Conflicting Interests and Environmental Education - The MUVIN Programme. Copenhagen: Research Programme for Environmental and Health Education, Department of Curriculum Research, Danish School of Education.

Cheng, J., \& Monroe, M. (2012). Connection to nature: Children's affective attitude toward nature. Environment and Behavior, 44, 31-49. doi:10.1177/0013916510385082

Chin, W. (1998). The Partial Least Squares approach to Structural Equation Modelling. In G.A. Marcoulides, Modern Methods for Business Research (pp. 295-336). New York: Tylor and Francis Group.

Collins, J. (2011). Good to Great: Why Some Companies Make the Leap... And Others Don't. New York: Harper Collins Publishers.

Corral-Verdugo, V., Frías, M., \& García, C. (2010). Introduction to the psychological dimensions of sustainability. Psychological approaches to sustainability, 3-18.

Dussan, E. (2012). Alineación de los valores individuales y corporativos y propuesta de intervención (Proyecto de grado para optar al título de maestría en administración de empresas MBAProfesional). Universidad del Norte, Barranquilla, Colombia.

Esposito, V., Trinchera, L., Squillacciotti, S., \& Tenenhaus, M. (2008). REBUS-PLS: A responsebased procedure for detecting unit segments in PLS path modelling. Applied Stochastic Models in Business and Industry, 24(5), 439-458.

Falk, R., \& Miller, N. (1992). A primer for soft modeling. Akron, O H: University of Akron Press.

Froján, M.X., Alpañes, M., Calero, A., \& Vargas, I. (2010). Una concepción conductual de la motivación en el proceso terapéutico. Psicothema, 22(4), 556-561.

Galvis, A.H. (2009). Influencia del núcleo familiar en la formación ambiental del niño-niña. Estudio de caso: Institución preescolar liceo infantil Casita Encantada. Localidad Barrios Unidos. Bogotá, D.C. (Trabajo de investigación para optar al grado de magíster en gestión ambiental). Pontificia Universidad Javeriana, Facultad de estudios Ambientales, Colombia. 
Guzmán, A., \& Fernández, G. (2013). Educación ambiental y problemas ambientales globales: ¿es posible el cambio de actitudes y comportamiento socioambiental? Revista Electrónica do Mestrado em Educacão Ambiental, 30(1), 252-263.

Hair, J.F., Anderson, R.E., Tatham R.L., \& Black, W. (1995). Análisis Multivariante (5a ed.). España: Pretince Hall.

Henseler, J., Ringle, C.M., \& Sinkovics, R.R. (2009). The Use of Partial Least Squares Path Modeling in International Marketing. Advances in International Marketing, 20(2), 277-319.

Heyl, M.E. (2012). Actitudes y conductas ambientales de los alumnos de la escuela de ingeniería de la PUC (Tesis para optar al grado de Magíster en Ciencias de la Ingeniería). Pontificia Universidad Católica de Chile, Santiago, Chile.

Hollman, V. (2012). El humor gráfico y la educación de la mirada ambiental, Revista Sociedade \& Natureza, 24(2), 227-242.

Hrebiniak, L. (2013). Making strategy Work: Leading Effective Execution and Change (2a ed.). United States of America: Pearson Education, Inc.

Jensen, B., \& Schnack, K. (2006). The Action Competence Approach in Environmental Education. Environmental Education Research, 12(3-4), 471-486.

López, M.J. (2010). Actitudes ambientales de la población de la localidad de Mar Chiquita hacia la conservación de la reserva de biosfera Parque Atlántico Mar Chiquito (Informe Final Beca MAB 2010). Universidad Nacional de Mar del Plata, Argentina.

López, P. (2010). Variables Asociadas a la Gestión Escolar Como Factores de Calidad Educativa. Estudios Pedagógicos, 36(1), 147-158.

Martí, J.J., Martí-Vilar, M., \& Almerich, G. (2014). Responsabilidad social universitaria: influencia de valores y empatía en la autoatribución de comportamientos socialmente responsables. Revista Latinoamericana de Psicología, 46(3), 160-168.

Martínez, M. (2012). El papel de la motivación auto-determinada en el entendimiento de actitudes e intenciones hacia la compra de productos ecológicos. Revista de Estudios Empresariales, 2, 96-115.

Matthies, E., Selge, S., \& Klöckner, C. (2012). The role of parental behavior for the development of behavior specific environmental norms - The example of recycling and re-use behavior. Journal of Environmental Psychology, 32(3), 277-284.

Medina, I., \& Páramo, P. (2014). La investigación en educación ambiental en América Latina: un análisis bibliométrico. Revista Colombiana de Educación, 66, 19-72.

Méndez, C., \& Rondón, M. (2012). Introducción al análisis factorial exploratorio. Revista colombiana de psiquiatría, 41(1), 197-207.

Ministerio del Medio Ambiente, Gobierno de Chile. (2010). Educación Ambiental y Desastres Naturales. Revista Educación Ambiental, 8(12). Recuperado desde http://portales.mineduc.cl/ usuarios/convivencia_escolar/doc/201205231613040.RevistaEA12junio2010.pdf

Ministerio del Medio Ambiente, Gobierno de Chile. (2013). Comportamiento Ambiental de la Ciudadanía. Informe Final. Santiago de Chile. Recuperado desde http://www.mma.gob.cl/1304/ articles-55920_AnexoE.pdf

Miranda, L.M. (2013). Cultura ambiental: un estudio desde las dimensiones de valor, creencias, actitudes y comportamientos ambientales. Producción + Limpia, 8(2), 94-105.

Moyano, E., Encina, Y., \& Vicente, D. (2007). Evaluación del Sistema Nacional de Certificación Ambiental de Establecimientos Educacionales (SNCAE) en Chile: Operatoria e impacto. Revista Electrónica Internacional de la Unión Latinoamericana de Entidades de Psicología, 10.

Müderrisoğlu, H., \& Altanlar, A. (2011). Attitudes and behaviors of undergraduate students toward environmental issues. International Journal of Environmental Science and Technology, 8(1), 159-168.

Murray, P. (2011). The Sustainable Self: A Personal Approach to Sustainability Education. London: Earthscan Ltda. 
Niven, P. (2002). Balanced Scorecard Step-by-Step: Maximizing Performance and Maintaining Results. New York: John Wiley \& Sons, Inc.

Obregón, E. (2011). Cultura Organizacional basada en Valores como Medio de Fortalecimiento de las Empresas Corporativas (Tesis de Maestría). Universidad Panamericana, Facultad de Ciencias Económicas, Guatemala.

Oviedo, H.C., \& Campo-Arias, A. (2005). Aproximación al uso del coeficiente Alfa de Cronbach. Revista colombiana de psiquiatría, 34(4), 572-580.

Penas, S. (2008). Aproximación a los valores y estilos de vida de los jóvenes de 13 y 14 años de la provincia de La Coruña (Tesis Doctoral). Universidad de Santiago de Compostela, Facultad de Psicología, Santiago de Compostela.

Pérez, D. (2013). Comportamiento ambiental en estudiantes de secundaria. Revista Vinculando. Recuperado desde http://vinculando.org/, Categoría: Educación.

Prieto, M. (2011). Actitudes y Valores. Innovación y Experiencias Educativas, 41, 1-8 Recuperado desde https://www.csif.es/contenido/andalucia/educacion/91934

Quintero, C.M. (2010). La cultura organizacional y los aspectos que la llevan a ser fuente del incumplimiento del direccionamiento estratégico de las empresas (Repositorio Institucional UMNG: Universidad Militar Nueva Granada, Bogotá). Recuperado desde http://hdl.handle. net/10654/3698

Reyes, H., \& Cardona, L. C. (2015). La educación ambiental como estrategia necesaria para la planificación de nuevos enfoques regionales en el departamento del Meta. Revista Sophia, 11(2), 169-184.

Rubín, K. (1998). Social and Emotional Development from a Cultural Perspective. Developmental Psychology, 34(4), 611-615.

Sánchez, M., Garza-González, A., \& Rodríguez, M. (2014). Análisis de la percepción y conducta ambiental mediante una red bayesiana. Revista de Psicología Universidad de Chile, 23(2), 56-70.

Sandoval, M. (2012). Comportamiento sustentable y educación ambiental: una visión desde las prácticas culturales. Revista Latinoamericana de Psicología, 44(1), 181-196.

Secretaría de Ambiente y Desarrollo Sustentable de la Nación. (2012). Educación Ambiental: Ideas y propuestas para docentes Nivel secundario. Argentina: Autor.

Stern, P.C. (1999). A value-belief-norm theory of support for social movements: The case of environmentalism. Human ecology review, 6(2), 81-98.

Tsevreni, I. (2011). Towards an environmental education without scientific knowledge: an attempt to create an action model based on children's experiences, emotions and perceptions about their environment. Environmental Education Research, 17(1), 53-67.

Zarrintaj, A., Sharifah, Z., Binti, Z., \& Abdul, S. (2011). Influence of Age and Level of Education on Environmental Awareness and Attitude: Case Study on Iranian Students in Malaysian Universities. The Social Sciences, 6(1), 15-19.

\section{AGRADECIMIENTOS}

Los autores agradecen el apoyo entregado por la Universidad de La Serena para terminar exitosamente esta investigación. 